\title{
Diffusion of Bacteriophage Capsids on a Glass Surface during Single- Particle Fluorescence Microscopy
}

\author{
P. Serwer, I. Wu, S. Huang and G. A. Griess \\ Dept. Biochemistry, The University of Texas Health Sci. Ctr, San Antonio, TX 78229-3900
}

To increase the rate of collision during diffusion, restriction of diffusion to a surface (i.e., diffusion in two dimensions) is an alternative to diffusion in three dimensions. The surface might be a cellular membrane [1,2]. Diffusion in two dimensions is also useful for bypassing limitations of single-particle biochemical analysis in three dimensions by fluorescence microscopy. These limitations include diffusion that is either (a) so rapid that a diffusing particle cannot be visualized, or (b) out of the plane of focus. A previous study [3] found that bacteriophage T7 capsids on a glass surface undergo diffusion in two dimensions during single-particle fluorescence microscopy. The present study analyzes this diffusion.

Diffusion in two dimensions was observed for Alexa 488-labeled T7 capsids called MLD capsid II [3]. Three diffusing particles and one immobile particle are shown in the train of images in Figure 1 (time between images, $t_{d},=0.033$ seconds). Dashed lines in Figure 1 intersect at the immobile particle. To determine an effective diffusion constant $\left(D_{\mathrm{E}}\right)$ for a single diffusing particle, the mean square displacement was measured for a fixed $t_{d}[3]$. However, $D_{\mathrm{E}}$ in the $\mathrm{X}$-direction $\left(D_{\mathrm{EX}}\right)$ was sometimes (but not always) different from $D_{\mathrm{E}}$ in the $\mathrm{Y}$-direction $\left(D_{\mathrm{EY}}\right)$, when measured on a single diffusing capsid (Figure 2; squares indicate $D_{\mathrm{EX}}$; circles indicate $D_{\mathrm{EY}}$; empty symbols are for one particle, filled symbols for another). Results differed significantly among different capsids in the same field. However, the following trends were observed: (a) Both $D_{\mathrm{EX}}$ and $D_{\mathrm{EY}}$ (for a single capsid) decreased as a function of $t_{d}$, for $t_{d}$ above $0.1 \mathrm{sec}$. (b) The $D_{\mathrm{E}}$ was constant for smaller values of $t_{\mathrm{d}}$.

The data of Figure 2 appear to be the first analysis of diffusion for a single particle on a glass surface. The mechanism is not known for a capsid's apparent diffusion in two dimensions only. The $D_{\mathrm{E}}$ 's extrapolated to $\mathrm{t}_{\mathrm{d}}=0$ are an order of magnitude smaller than the $D$ determined for the same capsid diffusing in three dimensions [3]. The anisotropy of diffusion is possibly explained by either (a) anisotropy of the glass surface, or (b) anisotropy of the capsid if the capsid was not spinning. The decrease of $D$ with increasing $t_{d}$ is expected for confined, but not unconfined, diffusion in two dimensions [4]. Yet, the authors observed no confinement. In any case, diffusion in two dimensions should be useful for the analysis of single biochemical events by fluorescence microscopy.

[1] G. Adam, M. Delbruck, Structural Chemistry and Molecular Biology, W. H. Freeman and Co., San Francisco, 1968, pp. 198-215.

[2] A. Pralle et al., J. Cell Biol. 148 (2000) 997.

[3] S. Huang, S.J. Hayes and P. Serwer, J. Struct. Biol. 135 (2001) 270.

[4] M. J. Saxton, Curr. Topics Membr. 48 (1999) 229.

[5] This work was supported by NIH (GM24365) and the Welch Foundation (AQ-764). 


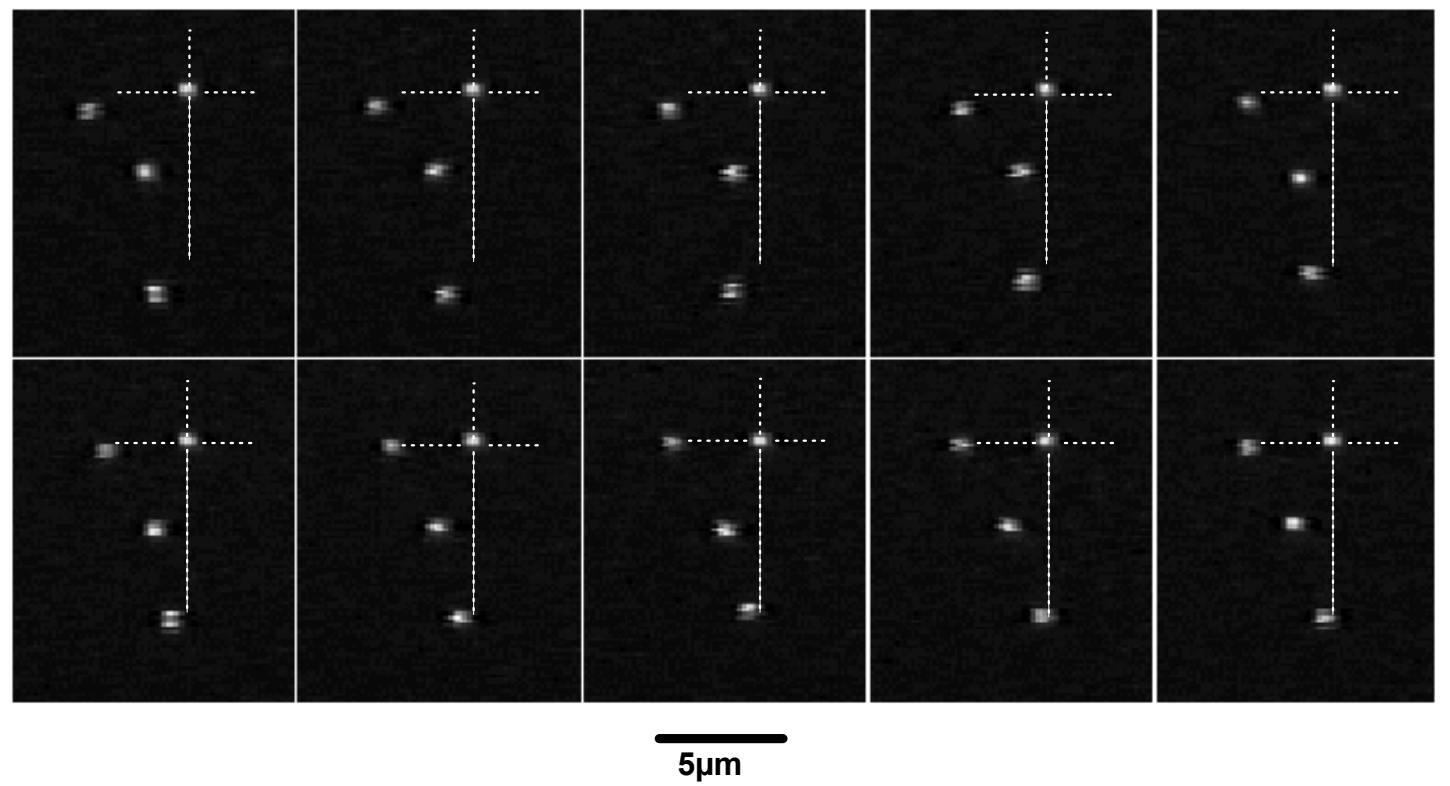

Figure 1. Bacteriophage T7 capsids undergoing diffusion in two dimensions. The sample is Alexa 488-labeled T7 MLD capsid II (3) placed between a cover glass and a glass slide. The buffer is $0.01 \mathrm{M}$ Tris-Cl, pH 7.4, 0.001M EDTA, $100 \mu \mathrm{g} / \mathrm{ml}$ gelatin, $1 \%$ ß-mercapto-ethanol, $0.02 \%$ Triton X-100.

Fluorescence microscopy of the surface of the cover glass was performed with analogue video recording (3). The analogue video was converted to digital video for both analysis and presentation. The dashed axes are centered on the only particle that is motionless. These axes are a reference for observing the motion of the other particles. The time increases left-to-right, top-to-bottom.
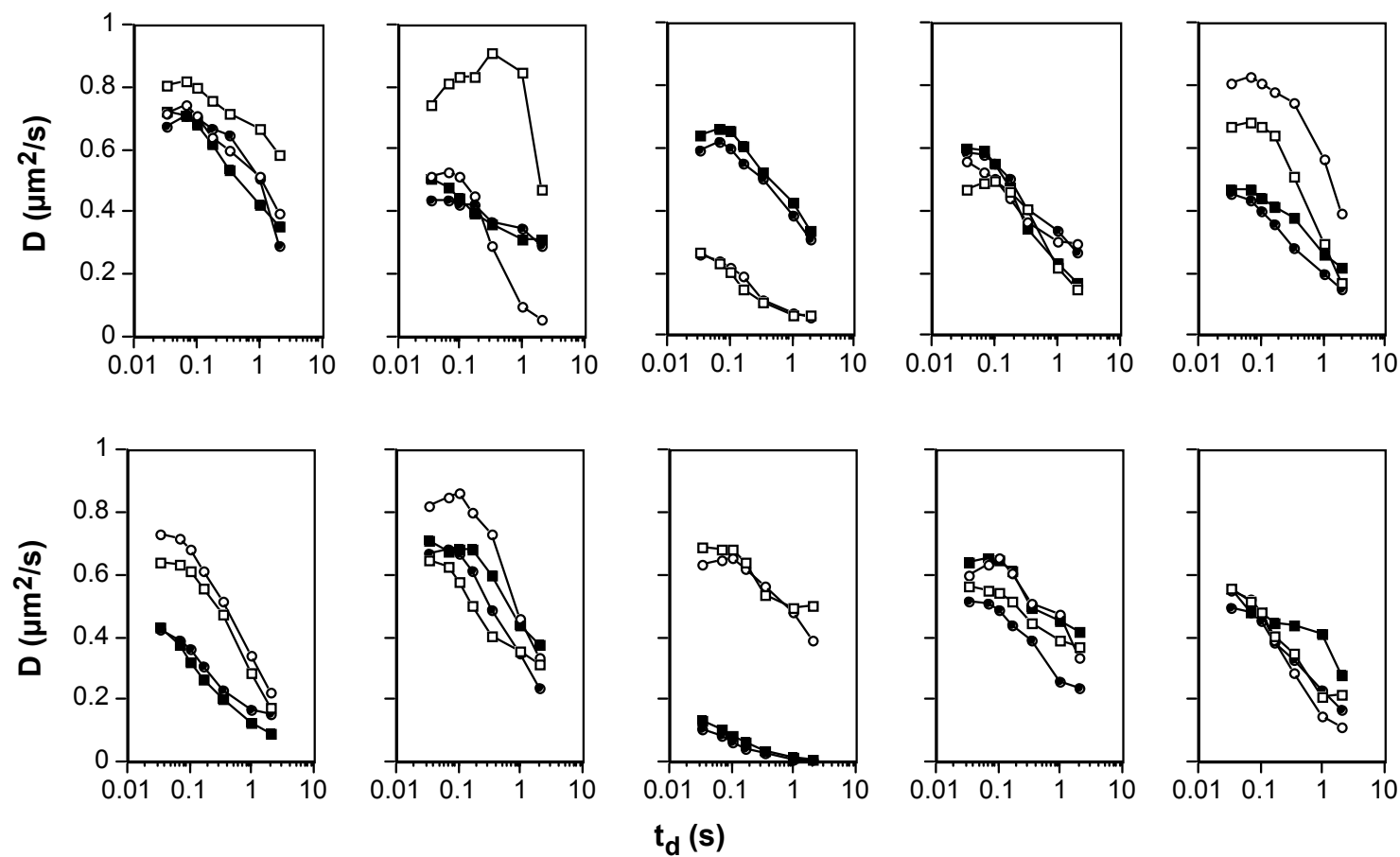

Figure 2. Plots of $D_{E X}$ and $D_{E Y}$ as a function of $t_{d}$. Each row has five sets of plots from a single field (two fields). The squares represent $\mathrm{D}_{\mathrm{EX}}$ and the circles represent $\mathrm{D}_{\mathrm{EY}}$. The filled symbols represent one particle; the empty symbols represent another. That is, data for two particles are in each panel. 